\title{
Underreporting of meningococcal disease incidence in the Netherlands: Results from a capture-recapture analysis based on three registration sources with correction for false positive diagnoses
}

\author{
Sabine C. de Greeff ${ }^{1}$, Lodewijk Spanjaard ${ }^{2}$, Jacob Dankert ${ }^{2}$, Christian J.P.A. Hoebe ${ }^{3}$, Nico \\ Nagelkerke ${ }^{4}$ \& Hester E. de Melker ${ }^{1}$ \\ ${ }^{1}$ Centre for Infectious Disease Epidemiology, National Institute for Public Health and the Environment, Bilthoven, The \\ Netherlands; ${ }^{2}$ Netherlands Reference Laboratory for Bacterial Meningitis, Academic Medical Centre, Amsterdam, The \\ Netherlands; ${ }^{3}$ Eastern South Limburg Municipal Public Health Service, Heerlen, The Netherlands; ${ }^{4}$ Computerization and \\ Methodological Consultancy Unit, National Institute of Public Health and the Environment, Bilthoven, The Netherlands
}

Accepted in revised form 9 February 2006

\begin{abstract}
In order to come to a reliable evaluation of the effectiveness of the chosen vaccination policy regarding meningococcal disease, the completeness of registrations on meningococcal disease in the Netherlands was estimated with the capture-recapture method.Data over 1993-1998 were collected from (A) mandatory notifications $(n=2926)$; $(\mathrm{B})$ hospital registration $(n=3968) ; \quad(C)$ laboratory surveillance $(n=3484)$. As the standard capture-recapture method does not take into account false positive diagnoses, we developed a model to adjust for the lack of specificity of our sources.We estimated that 1363 cases were not registered in any of the three sources in the period of study. The completeness of the three sources was therefore estimated at $49 \%$ for source A, $67 \%$ for source B and $58 \%$ for source C. After
\end{abstract}

adjustment for false positive diagnoses, the completeness of source $\mathrm{A}, \mathrm{B}$, and $\mathrm{C}$ was estimated as $52 \%, 70 \%$ and $62 \%$, respectively. The capture-recapture methods offer an attractive approach to estimate the completeness of surveillance sources and hence contribute to a more accurate estimate of the disease burden under study. However, the method does not account for higher-order interactions or presence of false positive diagnoses. Being aware of these limitations, the capture-recapture method still elucidates the (in)completeness of sources and gives a rough estimate of this (in)completeness. This makes a more accurate monitoring of disease incidence possible and hence attributes to a more reliable foundation for the design and evaluation of health interventions such as vaccination programs.

Key words: capture-recapture, correction for false-positives, log-linear model, meningococcal disease

\begin{abstract}
Abbreviations $\mathrm{AIC}=$ Akaike Information Criterion; $\mathrm{df}=$ Degree of freedom; ICD $=$ International Classification of Diseases; IGZ = Inspectorate of Health Care; MHS = Municipal Health Service; NMR = National Medical Registration; NRBM = National Reference Laboratory for Bacterial Meningitis; SAS = Statistical Analysis System
\end{abstract}

\section{Introduction}

Meningococcal disease is endemic worldwide [1, 2]. In the Netherlands the incidence of meningococcal disease has previously been reported to be approximately 3-4 per 100,000/year [3]. Serogroup B and more recently $\mathrm{C}$ are the most common serogroups [4]. Because of the emergence of serogroup $\mathrm{C}$ a national vaccination campaign against serogroup $\mathrm{C}$ was launched for all 1-18 year-old in the Netherlands in 2002. In addition, from September 2002 onwards vaccination at the age of 14 months was included in the routine childhood vaccination program [5]. Monitoring the incidence of meningococcal disease is essential to evaluate the effectiveness of the chosen vaccination strategy, and to advice on the use of vaccines against serogroup $\mathrm{B}$ when they become available.

Three data sources are available for monitoring meningococcal disease in the Netherlands: a mandatory notification system, a national hospital registration and a laboratory surveillance system. In this study we applied the capture-recapture method to estimate the completeness of these three surveillance sources [6]. Knowledge on the completeness (coverage or sensitivity) of these sources will contribute to the improvement of our existing surveillance system and hence makes more accurate monitoring of the true disease burden possible.

Since we have three sources we were able to adjust for dependencies between sources using log-linear modeling. With the method the number of cases not 
registered in any of the sources, for instance because these cases were incorrectly diagnosed (false negatives), can be estimated. However, the standard capture-recapture method does not take into account "contamination" of the sources by false positive errors in disease diagnosis, which could occur by incorrect diagnoses or clerically mistakes in registration. Such false positives would lead to an overestimation of the disease burden and may invalidate the application of statistical methods that assume absence of these type of errors. Therefore, we developed a model to adjust for this lack of specificity of sources.

\section{Material and methods}

\section{Data sources}

Cases of meningococcal disease over the period 1993-1998 were collected from three sources: mandatory notifications (source A), hospital registration (source B) and laboratory surveillance (source C).

Data on notified cases (A) were obtained from the Inspectorate of Health Care (IGZ). According to the Dutch Communicable Disease Act, physicians have to report each case of invasive meningococcal infection within $24 \mathrm{~h}$ to their Municipal Public Health Service (MHS). After verification by the MHS the case will be reported to the IGZ. The notification database contains: an unique patient number, date of birth, gender, initial of first and last name, postal code, municipality, date of notification, date of first symptoms, date of diagnosis, and age at notification date.

Hospital episodes (B) of meningococcal disease were extracted from the National Medical Registration (NMR). Diagnoses were recorded using the 9th International Classification of Diseases based on clinical diagnoses. Cases with code 0360 (meningococcal meningitis), 0362 (meningococcal septicemia) and 0363 (meningococcal syndrome of WaterhouseFriderichsen) as main diagnosis, were selected. Data in this source are: unique patient number, date of birth, gender, postal code, municipality, date of hospital admission, ICD-code and outcome (deceased or not).

The Netherlands Reference laboratory for Bacterial Meningitis (NRBM) collects meningococcal strains from blood and/or CSF isolates of patients with meningococcal disease. Clinical microbiological laboratories throughout the country send these materials voluntarily to NRBM. This source (C) contains: an unique patient number, date of birth, gender, initial of first and last name, municipality, laboratory that submitted the strain, date of collection and receipt of the sample, and typing results.

\section{Data linkage between sources}

Records were linked to assess the overlap between sources. For reasons of confidentiality all three data sources lack the names of patients. Therefore, linkage of records had to be done by other patient characteristics. First, records between two sources were linked by three to four patient variables (date of birth, gender, postal code/place of residence and initials) and afterwards by the various combinations of two to three of these variables. The first linkages by three to four variables were considered correct. All further linkages were verified manually and - if plausible regarding all variables - assumed to be correct. After linkage between two sources (A and B, $\mathrm{A}$ and $\mathrm{C}, \mathrm{B}$ and $\mathrm{C}$ ) linked records were linked within the three sources.

\section{Capture-recapture estimates}

The capture-recapture method uses the degree of overlap between sources to estimate the number of cases not registered in any of the sources and hence the total number of cases. For example, with two sources three frequencies can be observed: cases registered only in source $1\left(n_{1}\right)$, cases only in source 2 $\left(n_{2}\right)$ and cases registered in both sources $\left(n_{12}\right)$. One frequency is only estimable: the cases not registered in any of the sources $(x)$. The magnitude of the latter $(x)$ can be estimated by $x=\left(n_{1} * n_{2}\right) / n_{12}$. And the total population size $(N)$ can than be calculated by $N=n_{1}+n_{2}+n_{12}+x$.

Application of this method assumes: (1) a closed population, (2) individuals can be matched from one source to another (3) each individual has the same chance of being included in the sources, (4) and, for two sources, independence between these sources [7]. Such independence, or lack of interaction, implies that the probability of being registered in one source is the same for cases registered in a second source and those who are not. For three sources, existence of interaction can be tested by comparing the twosource capture-recapture estimates and by calculating the Odds Ratios between two sources within the third [7-10]. For three sources, pair-wise independence of sources is not required, and can be adjusted using log-linear models. In fact, with three sources, different models based on different assumptions on the (in)dependence structure of the data, can be fitted and compared. However, the highest, three-way, interaction is not identifiable and has to be assumed absent [6, 10]. Three-way interactions would be logical, for example, if some cases are highly likely to be either included or missed by all three sources. Models were compared by the likelihood ratio test statistic $\left(G^{2}\right)$ and by the Akaike Information Criterion $\left(\mathrm{AIC}=G^{2}-2(\mathrm{df})\right)[6]$. The best fitting model was the one with the lowest $G^{2}$ and AIC. Confidence intervals were calculated as proposed by Bishop et al. [11]. 
Sensitivity of each source was estimated dividing the observed number of cases in each source by the capture-recapture estimate of the total population. The Statistical Analysis System (SAS) version 9.1.3. was used to perform the analyses.

The capture-recapture method assumes that there are no false positives due to misdiagnoses or incorrect registration [12]. However, in our study this assumption may not be entirely correct. Some false positive cases were expected to be present in source B (e.g. a hospitalized case with an incorrectly registered discharge diagnosis of meningococcal disease) and to be age-dependent [13]. Also, though to a lesser degree, false positives were expected to be present in source A (e.g. a notified patient with meningitis or septicemia caused by another pathogen (Streptococcus pneumoniae, Haemophilus influenzae) mistaken for a case of invasive meningococcal disease). In source $\mathrm{C}$ we did not expect false positives as they were all laboratory confirmed. All records registered in two (or more) sources were counted as true positives.

To account for the false positives we assumed that log-linear parameters would not depend on age in the absence of false positives. This seems reasonable as meningococcal disease is so severe that you expect all true patients - irrespective of age - to show up either at the doctor or in the hospital. By contrast, the false positive error rate was hypothesized to depend on age. We assumed that the false positive error rate depends on the overall probability of hospital admission (implying severe disease) - i.e. the more chance being severely ill in general, the bigger the chance of incorrect registration in a health registerand not on other factors. Since the chance for hospitalization is age dependent, the probability of being admitted to the hospital for any cause by age-category (calculated as the mean chance for hospitalization in this period i.e. $0-19$ years: $4 \%, 20-44$ years: $5 \%$, 45-64 years: $8 \%, 65$ and older: $14 \%$ ) [14] was used to estimate the extent of "contamination" by false positive diagnoses. To estimate the number of false positives we categorized all patients into the four age groups, and assumed that for each age group the $2 \times 2 \times 2$ table of cell entries was the sum of a $2 \times 2 \times 2$ table of false positives and a $2 \times 2 \times 2$ table of true positives. The $2 \times 2 \times 2$ table of false positives were hypothesized to consist of zeros with the exception of cells corresponding to only source B and only source A. These cells were assumed to have entries proportional to the age-group specific probability of hospitalization, the total number in that age group, and source (A or B) specific "false-positivity" parameters. Approximate maximum likelihood estimates of the 2 false positivity parameters and 7 true positivity parameters (specifying the structure $2 \times 2 \times 2$ table of true positives) were obtained by weighted linear regression using SAS PROC NLIN.

Estimates of (age-specific) false positives were then subtracted from initial cell counts. After this adjustment for false positives new capture-recapture point-estimates were calculated with the SAS GENMOD-procedure.

\section{Results}

From 1993 to 1998,4599 cases had been recorded in at least one of the three sources. Of these 2234 were identified in the three sources, 314 were matches between source A and B, 189 were matches between source $\mathrm{A}$ and $\mathrm{C}$ and 808 were matches between source $\mathrm{B}$ and $\mathrm{C}$ (Figure 1).

Pair-wise capture-recapture estimated 4557 cases for source A and B, and 4545 for source B and C. The two-source capture-recapture estimate for source A and $\mathrm{C}$ amounted 4207 patients, substantially lower than the other two estimates (Table 1). This lower estimate suggests positive dependency between source $\mathrm{A}$ and $\mathrm{C}$, with among the cases registered within source B an Odds Ratio between source A and source $\mathrm{C}$ of 5.38 (95\% CI: 4.6-6.3). The Odds Ratio between source B and C within source A was 7.11 (95\% CI: 5.6-9.0), and the Odds Ratio between source A and B among cases registered in source $\mathrm{C}$ was 3.70 (95\% CI: 3.0-4.5).

Log-linear models were used to account for dependencies between data sources. Based on the numbers shown in Figure 1, eight different loglinear models were used to estimate the cases not registered in any of the three sources. Estimates of the capture-recapture analysis with three sources are presented in Table 2. None of the non-saturated models fitted the data very well. The model with the smallest $G^{2}$ and AIC was the one with all three two-way interaction terms included. However, such a saturated model should be considered with skepticism as it always gives a $G^{2}=0$. Lack of degrees of freedom makes it impossible to test its implicit assumption of absence of higher (tree-way) interactions. Based on the model, 1363 cases in the period 1993-1998 were not registered in any of the

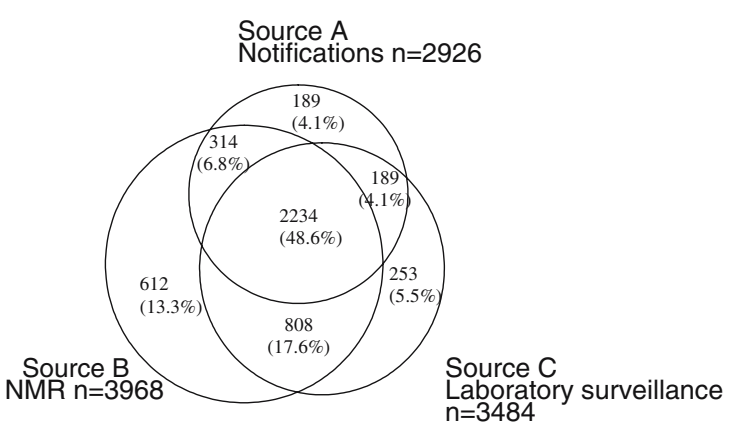

Figure 1. Distribution of cases of meningococcal disease in three registrations in the Netherlands from 1993 to 1998 , without correction for false-positives. 
Table 1. Two-source capture recapture estimates of numbers of patients with meningococcal disease in the Netherlands, 1993-1998

\begin{tabular}{lllllllll}
\hline Source 1 & Source 2 & $n_{\text {source 1 }}{ }^{\mathrm{a}}$ & $n_{\text {source 2 }}{ }^{\mathrm{a}}$ & $n_{\text {source 1, source 2 }}{ }^{\mathrm{a}}$ & $\begin{array}{l}N_{\text {cap-recap }} \\
\left(95 \% \mathrm{CI}^{\mathrm{c}}\right)\end{array}$ & $\begin{array}{l}\text { Completeness } \\
\text { source 1 } \\
\left(95 \% \mathrm{CI}^{\mathrm{c}}\right)\end{array}$ & $\begin{array}{l}\text { Completeness } \\
\text { source 2 } \\
\left(95 \% \mathrm{CI}^{\mathrm{c}}\right)\end{array}$ \\
\hline Source A & Source B & 378 & 1420 & 2548 & $4557(4519-4595)$ & $64 \%(63-66)$ & $87 \%(86-88)$ \\
Source A & Source C & 503 & 1061 & 2423 & $4207(4169-4245)$ & $70 \%(68-70)$ & $83 \%(82-84)$ \\
Source B & Source C & 926 & 442 & 3042 & $4545(4517-4572)$ & $87 \%(86-89)$ & $77 \%(75-78)$ \\
\hline
\end{tabular}

Source $\mathrm{A}=$ notification system (MHS), Source $\mathrm{B}=$ hospital registration (NMR) and Source $\mathrm{C}=$ laboratory surveillance (NRBM).

${ }^{a}$ Number of meningococcal cases registered only in source 1, only in source 2 or in both sources.

${ }^{\mathrm{b}}$ Estimate of the total number of patients with meningococcal disease.

${ }^{\mathrm{c}} 95 \%$ confidence interval.

Table 2. Log-linear models fitted to three sources of data on meningococcal disease and the estimated number of meningococcal cases in the Netherlands in 1993-1998

\begin{tabular}{|c|c|c|c|c|c|c|c|c|c|c|c|c|}
\hline \multirow{2}{*}{$\frac{\text { Model }}{1}$} & \multicolumn{3}{|c|}{$\begin{array}{l}\text { Main } \\
\text { terms }\end{array}$} & \multicolumn{3}{|c|}{$\begin{array}{l}\text { Interaction } \\
\text { terms }\end{array}$} & \multirow{2}{*}{$\frac{G^{2 \mathrm{a}}}{596}$} & \multirow{2}{*}{$\frac{\mathrm{df}^{\mathrm{b}}}{3}$} & \multirow{2}{*}{$\begin{array}{l}N_{\text {cap-recap }}{ }^{\mathrm{c}} \\
\left(95 \% \mathrm{CI}^{\mathrm{d}}\right)\end{array}$} & \multirow{2}{*}{$\begin{array}{l}\text { Completeness } \\
\begin{array}{l}\text { Source A } \\
\left(95 \% \mathrm{CI}^{\mathrm{d}}\right)\end{array} \\
63 \%(61-64)\end{array}$} & \multirow{2}{*}{$\begin{array}{l}\text { Completeness } \\
\begin{array}{l}\text { Source B } \\
\left(95 \% \mathrm{CI}^{\mathrm{d}}\right)\end{array} \\
85 \%(84-86)\end{array}$} & \multirow{2}{*}{$\begin{array}{l}\text { Completeness } \\
\text { Source C } \\
\left(95 \% \mathrm{CI}^{\mathrm{d}}\right) \\
75 \%(73-76)\end{array}$} \\
\hline & A & $\mathrm{B}$ & $\mathrm{C}$ & & & & & & & & & \\
\hline 2 & A & B & $\mathrm{C}$ & $\mathrm{A}, \mathrm{B}$ & & & 581 & 2 & $4686(4664-4708)$ & $62 \%(61-64)$ & $85 \%(84-86)$ & $74 \%(70-73)$ \\
\hline 3 & A & B & $\mathrm{C}$ & $\mathrm{A}, \mathrm{C}$ & & & 346 & 2 & $4714(4686-4742)$ & $62 \%(61-63)$ & $84 \%(83-85)$ & $74 \%(73-75)$ \\
\hline 4 & A & B & $\mathrm{C}$ & $\mathrm{B}, \mathrm{C}$ & & & 548 & 2 & $4715(4687-4743)$ & $62 \%(61-63)$ & $84 \%(83-85)$ & $74 \%(73-75)$ \\
\hline 5 & A & B & $\mathrm{C}$ & A,B & $\mathrm{A}, \mathrm{C}$ & & 328 & 1 & $4791(4750-4832)$ & $61 \%(60-62)$ & $83 \%(82-84)$ & $73 \%(71-74)$ \\
\hline 6 & A & B & $\mathrm{C}$ & A,B & $\mathrm{B}, \mathrm{C}$ & & 482 & 1 & 4852 (4785-4919) & $60 \%(59-62)$ & $82 \%(81-83)$ & $72 \%(71-73)$ \\
\hline 7 & A & B & $\mathrm{C}$ & $\mathrm{A}, \mathrm{C}$ & $\mathrm{B}, \mathrm{C}$ & & 172 & 1 & $4968(4885-5049)$ & $59 \%(58-60)$ & $80 \%(79-81)$ & $70 \%(69-71)$ \\
\hline 8 & A & $\mathrm{B}$ & $\mathrm{C}$ & A,B & $\mathrm{A}, \mathrm{C}$ & $\mathrm{B}, \mathrm{C}$ & 0 & 0 & $5962(5581-6343)$ & $49 \%(48-50)$ & $67 \%(65-68)$ & $58 \%(57-60)$ \\
\hline
\end{tabular}

$\mathrm{A}=$ notification system $(\mathrm{MHS}), \mathrm{B}=$ hospital registry $(\mathrm{NMR})$ and $\mathrm{C}=$ laboratory surveillance $(\mathrm{NRBM})$.

${ }^{a}$ Likelihood ratio statistic.

${ }^{\mathrm{b}}$ Degree of freedom.

${ }^{c}$ Estimate of the total number of patients with meningococcal disease.

${ }^{\mathrm{d}} 95 \%$ confidence interval.

three sources. The total number of cases with meningococcal disease in 1993-1998 would therefore amount to 5962 and the completeness of the three sources was estimated at $49 \%$ for source A, $67 \%$ for source B and $58 \%$ for source C.

Stratification by age-group showed that estimated completeness of surveillance sources decreased with increasing age-group (Figure 2). This age-dependency is inconsistent with the assumption that the log-linear parameters do not depend on age, and hence offers evidence for the existence of false-positive cases. Using weighted linear regression it was estimated that $9 \%$ of source $\mathrm{A}$ and $12 \%$ of source $\mathrm{B}$ were false-positives. After correction for these false-positive diagnoses by deleting them by age category, we estimated that 1074 patients were not registered in any of the three sources. Therefore, applying the model with all three two-source interactions, the total number of cases was estimated at 5577 in 1993-1998 and completeness was calculated $52 \%$ for source A, $70 \%$ for source $\mathrm{B}$ and $62 \%$ for source $\mathrm{C}$ (Table 3 ).

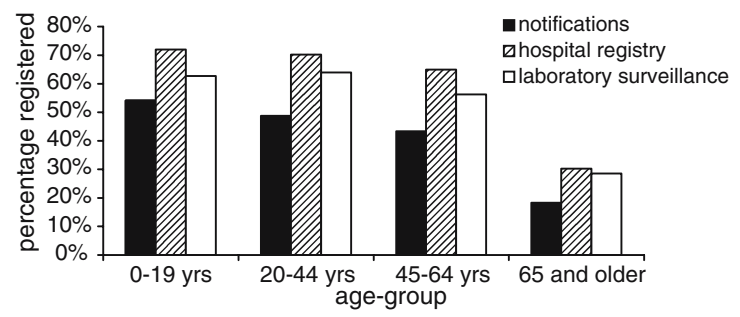

Figure 2. Completeness of notification system (black), hospital registry (shaded) and laboratory surveillance (white) for meningococcal disease, by age-group in the Netherlands, 1993-1998, without correction for false-positives.

\section{Discussion}

Capture-recapture methods are a unique tool to estimate the completeness of registrations. Provided several data-sources are available and records can be linked between them, the number of unregistered cases can be estimated. These conditions were met for meningococcal disease in the Netherlands in the 
Table 3. Log-linear models fitted to three sources of data on meningococcal disease and the estimated number of meningococcal cases in the Netherlands in 1993-1998, corrected for false positives

\begin{tabular}{|c|c|c|c|c|c|c|c|c|c|c|c|c|}
\hline \multirow{2}{*}{$\frac{\text { Model }}{1}$} & \multicolumn{3}{|c|}{$\begin{array}{l}\text { Main } \\
\text { terms }\end{array}$} & \multicolumn{3}{|c|}{$\begin{array}{l}\text { Interaction } \\
\text { terms }\end{array}$} & \multirow{2}{*}{$\frac{G^{2 \mathrm{a}}}{508}$} & \multirow{2}{*}{$\frac{\mathrm{df}^{\mathrm{b}}}{3}$} & \multirow{2}{*}{$\frac{N_{\text {cap-recap }}{ }^{\mathrm{c}}}{4560}$} & \multirow{2}{*}{$\begin{array}{l}\text { Completeness } \\
\text { source A (\%) } \\
64\end{array}$} & \multirow{2}{*}{$\begin{array}{l}\text { Completeness } \\
\text { source B }(\%)\end{array}$} & \multirow{2}{*}{$\begin{array}{l}\text { Completeness } \\
\text { source C (\%) } \\
76\end{array}$} \\
\hline & A & B & $\mathrm{C}$ & & & & & & & & & \\
\hline 2 & A & B & $\mathrm{C}$ & $\mathrm{A}, \mathrm{B}$ & & & 486 & 2 & 4582 & 63 & 85 & 76 \\
\hline 3 & A & B & $\mathrm{C}$ & $\mathrm{A}, \mathrm{C}$ & & & 293 & 2 & 4601 & 63 & 85 & 76 \\
\hline 4 & A & B & $\mathrm{C}$ & $\mathrm{B}, \mathrm{C}$ & & & 466 & 2 & 4603 & 63 & 85 & 76 \\
\hline 5 & A & B & $\mathrm{C}$ & A,B & $\mathrm{A}, \mathrm{C}$ & & 283 & 1 & 4669 & 62 & 83 & 75 \\
\hline 6 & A & B & $\mathrm{C}$ & A,B & $\mathrm{B}, \mathrm{C}$ & & 391 & 1 & 4731 & 61 & 82 & 74 \\
\hline 7 & A & B & $\mathrm{C}$ & $\mathrm{A}, \mathrm{C}$ & $\mathrm{B}, \mathrm{C}$ & & 167 & 1 & 4797 & 61 & 81 & 73 \\
\hline 8 & A & B & $\mathrm{C}$ & A,B & $\mathrm{A}, \mathrm{C}$ & $\mathrm{B}, \mathrm{C}$ & 0 & 0 & 5577 & 52 & 70 & 62 \\
\hline
\end{tabular}

$\mathrm{A}=$ notification system (MHS), $\mathrm{B}=$ hospital registry (NMR) and $\mathrm{C}=$ laboratory surveillance (NRBM).

${ }^{\text {a }}$ Likelihood ratio statistic.

${ }^{\mathrm{b}}$ Degree of freedom.

${ }^{\mathrm{c}}$ Estimate of the total number of patients with meningococcal disease.

period 1993-1998. Unfortunately, for the period after 1999 such analyses are no longer feasible since a change in the Communicable Disease Act made linkage between sources by personal identifiers impossible.

In our two-source (pair-wise) capture-recapture analysis the estimate of the total number of meningococcal cases was lowest based on notifications (A) and laboratory surveillance (C). The lower estimate suggest positive dependency between notifications and laboratory surveillance $[6,7]$. The dependency was reflected by the high Odds Ratio we found between the source pairs and can be explained by their underlying process: notified cases are often confirmed by isolation or detection of Neisseria meningitidis by a laboratory. However, the high Odds Ratios between the other source pairs suggest also positive dependency between laboratory surveillance and hospital registry and, to a lesser extent, between notifications and the hospital registry.

With the three-source capture-recapture analysis pair-wise dependencies between sources were accounted for by interaction terms in the log-linear model. However, the method assumes absence of higher (3-way) interaction terms [15]. Since none of the models (i.e. models $1-7$, Tables 2 and 3 ) fitted the data very well, the best model was the saturated model including all the main terms and all two-source interaction terms. In view of the fact that we observed strong pair-wise interactions between all sources, a three-source interaction (assumed absent in our model) would seem plausible. If so, this implies that our results would underestimate the actual number of patients and consequently would overestimate the completeness of the three registries [16].

Conversely, including patients that are erroneously registered as meningococcal cases overestimates the true burden of meningococcal disease and underestimates completeness. Traditional capture-recapture methods assume that all sources have a sensitivity of $100 \%$ or less, and a specificity of $100 \%$. The latter certainly holds for the applications for which the method was originally developed, viz estimating sizes of free living animal populations, where misclassification of animals will be rare. However, in health registries clerical mistakes are easily made. For example, in a study by Ackman et al. the sensitivity of hospital records in 1991 in New York State was assessed [17]. They found that, although the hospital discharge files listed almost twice as many patients with a diagnosis of meningococcal disease as were reported to the Department of Health, in one-third of these records the diagnosis was incorrect. We expected false positive diagnoses in both the hospital registry (B) and to a lesser degree in the mandatory notifications (A) in our study. Adjusting for these false positive cases decreased the estimated number of patients not registered in any of the three sources substantially and hence completeness estimates increased; a finding that has been demonstrated before for two-source capture-recapture methods [12].

Similar bias could occur by errors in record linkage. However, since we have linked on three to four variables and linkages were checked manually such errors are probably rare.

The aim of this study was to ascertain the completeness of each register to enable more accurate monitoring of the incidence of meningococcal disease. Apparently, all sources have the disadvantage of incompleteness. Although physicians have to notify each case of meningococcal infection, we only identified $60 \%$ of the estimated cases in the notifications (source A). This agrees with results from a similar study from Spanjaard et al. [18], and emphasizes that exclusive reliance on notifications would seriously underestimate the total incidence of meningococcal disease in the Netherlands. Similarly, surveillance of meningococcal disease exclusively based on laboratory data from NRBM (source C) would not be complete enough as only culture confirmed cases are included in this source. Due to loss of material or impossibility to detect the causal micro-organism 
after early antibiotic administration, laboratory confirmation might not be available for all cases.

Still, completeness of a disease registry alone is no guarantee for an adequate description of disease incidence. Concerning the NMR (source B), erroneously registration may have affected the sensitivity. Apparently, no single registry is sufficiently comprehensive in terms of the patient data it contains [19]. In the Netherlands, surveillance of meningococcal disease based on hospital admissions (B) and laboratory surveillance $(\mathrm{C})$ would give the most complete information, including important data on outcome and serotyping. However, the data in both sources are not as timely available as notification data.

In conclusion, for policy making and disease control, e.g. regarding the cost-effectiveness or evaluation of a new vaccination, public health officials need to know the size of the targeted population. Although insight in the number of patients is gained by compulsory reporting to registrations, these registrations are often incomplete or inaccurate [17]. The capturerecapture methods offer an attractive approach to estimate true incidence rates and are much less expensive than classic approaches to case finding [20]. However, in the presence of several incomplete registrations, they have important limitations that should not be ignored [21]. First, they are based on the not-testable assumption of absence of higher-order interactions and second, they assume absence of false positive diagnoses. In our study we managed to overcome, to some extent, the second shortcoming. However, in the absence of a fourth registry there is no way the first limitation can be overcome. Being aware of these limitations, the capture-recapture method still elucidates the completeness of sources and gives a rough estimate of this completeness. Taking into account the completeness makes a more accurate monitoring of disease incidence possible and hence attributes to a more reliable foundation for the design and evaluation of health interventions such as vaccination programs.

\section{Acknowledgement}

The authors thank N.A. van Hest for critically reviewing the manuscript.

\section{References}

1. van Deuren $\mathrm{M}$, Brandtzaeg $\mathrm{P}$, van der Meer JWM. Update on meningococcal disease with emphasis on pathogenesis and clinical management. Clin Microbiol Rev 2000; 13(1): 144-166.

2. Rosenstein NE, Perkins BA, Stephens DS, Popovic T, Hughes JM. Meningococcal disease. N Engl J Med 2001; 344(18): 1378-1388.
3. Hoebe CJPA, de Melker HE, Spanjaard L, Dankert J, Nagelkerke N. Space-time cluster analysis of invasive meningococcal disease. Emerg Infect Dis 2004; 10: 1621-6.

4. Netherlands Reference Laboratory for Bacterial Meningitis. Bacterial meningitis in the Netherlands. Annual report 2001. Amsterdam: University of Amsterdam, 2002.

5. de Vries M, Dankert J, Ruijs H, Timen A, de Greeff S, de Melker $\mathrm{H}$. Universal vaccination against group $\mathrm{C}$ meningococci and pneumococci; advice from the Health Council of the Netherlands [Dutch]. Ned Tijdschr Geneeskd 2002; 146: 1562-1563.

6. Hook EB, Regal RR. Capture-recapture methods in epidemiology: methods and limitations. Epidemiol Rev 1995; 17(2): 243-264.

7. Wittes JT, Colton T, Sidel VW. Capture-recapture methods for assessing the completeness of case ascertainment when using multiple information sources. J Chronic Dis 1974; 27: 25-36.

8. Cormack RM. Problems with using capture-recapture in epidemiology: an example of a measles epidemic. J Clin Epidemiol 1999; 52(10): 909-914.

9. International Working Group for Disease Monitoring and Forecasting. Capture-recapture and multiplerecord systems estimation 1: history and theoretical development. Am J Epidemiol 1995; 142: 1047-1058.

10. Pezzotti P, Piovesan C, Zanella F, et al. Estimating the cumulative number of human immunodeficiency virus diagnoses by cross-linking from four different sources. Int J Epidemiol 2003; 32: 778-783.

11. Bishop YMM, Fienberg SE, Holland PW. Discrete Multivariate Analysis: Theory and Practice. Cambridge, Massachusetts: The Massachusetts Institute of Technology, 1989, 237 pp-245.

12. Brenner H. Effects of misdiagnoses on disease monitoring with capture-recapture methods. J Clin Epidemiol 1996; 49: 1303-1307.

13. Davison KL, Crowcroft NS, Ramsay ME, et al. Enhanced surveillance scheme for suspected meningococcal disease in five regional health authorities in England: 1998. Commun Dis Public Health 2002; 5(3): -205-212.

14. Statline Central Statistics Netherlands, Statistics Netherlands, Voorburg/Heerlen, 2003 Available from http://statline.cbs.nl/StatWeb/table.asp?TT $=2 \& \mathrm{LA}=$ $\mathrm{nl} \& \mathrm{DM}=\mathrm{SLNL} \& \mathrm{PA}=7042 \mathrm{mc} \& \mathrm{D} 1=322-325 \& \mathrm{D} 2=$ $13-18$.

15. Chao A, Tsay PK, Lin SH, Shau WY, Chao DY. The applications of capture-recapture models to epidemiological data. Statist Med 2001; 20: 3123-3157.

16. Hook EB, Regal RR. Accuracy of alternative approaches to capture-recapture estimates of disease frequency: internal validity analysis of data from five sources. Am J Epidemiol 2000; 152: 771-779.

17. Ackman DM, Birkhead G, Flynn M. Assessment of surveillance for meningococcal disease in New York stat, 1991. Am J Epidemiol 1996; 144: 78-82.

18. Spanjaard L, Bol P, Ekker W, Zanen HC. The incidence of bacterial meningitis in the Netherlands - a comparison of three registration systems, 1977-1982. J Infect 1985; 11: 259-268.

19. Davies LA. Assessing the value of different sources of information on meningococcal disease. Community Med 1989; 11(3): 239-246. 
20. Laska EM. The use of capture-recapture methods in public health. Bull World Health Organ 2002; 80(11): 845.

21. Papoz L, Balkau B, Lellouch J. Case counting in epidemiology: limitations of methods based on multiple data sources. Int J Epidemiol 1996; 25(3): 474-478.
Address for correspondence: S.C. de Greeff, National Institute of Public Health and the Environment, P.O. Box 1, 3720 BA Bilthoven, The Netherlands

Phone: + 31-30-274-2982; Fax: + 31-30-274-4409

E-mail: sabine.de.greeff@rivm.nl 